\title{
On the Selection of MAC Optimised Routing Protocol for VANET
}

\author{
Kanu Priya \\ Research Scholar, Department of CSE, Guru Nanak Dev University Regional Campus, Jalandhar, India. \\ E-mail: kanupriya2310@gmail.com \\ Jyoteesh Malhotra \\ Head of CSE \& ECE Department, Guru Nanak Dev University Regional Campus, Jalandhar, India. \\ E-mail: jyoteesh@gmail.com
}

\begin{abstract}
In today's era of modernization, the concept of smart vehicles, smart cities and automated vehicles is trending day by day. VANET (Vehicular Adhoc Network) has also been emerging as a potential applicant to enable these smart applications. Though VANET is very much similar to MANET (Mobile Adhoc Network) but VANET has more severe challenges as compared to MANET due to hostile channel conditions and high degree of mobility. So lot of work related to MAC and Network Layer need attention from the network designers. In this paper MAC Layer has been optimised in terms of Queue Size by using QoS Parameters namely Packet Collision Rate, Packet Drop Rate, Throughput Rate and Broadcast Rate. In doing so, simulative investigations have been done to find out optimum queue size. For this purpose various routing protocols namely DSDV, AODV, ADV and GOD have been considered and optimum queue length for each of these have been obtained. Further the most efficient routing protocol has also been identified. Moreover this paper also compares the performance of most efficient Routing Protocols selected in terms of QoS parameters for different MAC Interfaces.
\end{abstract}

Index Terms-DSDV (Destination Sequenced Distance Vector Routing), ADV (Advanced Distance Vector Routing), GOD (General Operation Directory), ITS (Intelligent Transport System), NCTUns.

\section{INTRODUCTION}

In today's era VANET (Vehicular Adhoc Network) is one of the emerging technologies that has its usage in specific area of adhoc networks. Several attributes are gaining importance day by day like smart cities, smart vehicles, automated highways and bridges etc. VANET is very well organised and an autonomous wireless adhoc network. Hence this area is gaining momentum in every pulse of time. VANET is prominent area of Intelligent Transport System (ITS) that permits the vehicles to communicate with other vehicles and roadside equipments wirelessly. Whenever an alarming situation like traffic congestion, road accidents, fire incidents etc. are encountered; vehicles wirelessly transmit this crucial information with all the vehicles within its communication range. Hence it permits road safety to the users. But still there is need of optimization in certain key areas of routing layer, physical layer and Mac layer. As the vehicles are increasing on roads and all have varying driving behavior this implies VANET as a very dynamic. In VANET the mobile nodes have to follow some restrictions regarding patterns of movement and traffic conditions. So routing protocols govern the moving behavior of vehicles hence it is the most challenging issue for the researchers to deal with. The routing protocols used in VANET should be highly reliable and must be capable of adapting to network load with minimum delay in data transmission. The main focus of this paper is the optimisation of MAC Layer as well as Routing Layer using IEEE 802.11a Interface. In order to evaluate the performance, extensive simulations have been performed. For doing so, Multi interface car is used by varying queue sizes in various routing protocols. Queues are basically used to store and forward the data packets. Various types of queues are present out of which FIFO queue is used here. Queue size is also an important parameter to measure the performance of routing protocols in VANETs. Queue size may be defined as the length of queue to be used for transferring data packets successfully to the destination. Considering queue size as a key objective this paper aims at selection of optimum queue length for various routing protocols. For achieving this routing protocols namely DSDV, AODV, ADV and GOD have been evaluated at different queue lengths on the basis of QoS parameters i.e. Packet Collision Rate, Packet Drop Rate, Throughput Rate and Broadcast Rate in city scenario. FIFO queue is implemented at five different queue sizes to determine the most appropriate queue length for different protocols. Further these performance parameters have been compared and analysed for finding the most efficient routing protocol for 802.11a interface. The simulations are performed in GUI based tool named as NCTUns (National Chiao Tung University) 6.0 over a simulation time of 400seconds and results have been achieved in the form of graphs presented in IV Section.

The rest of the paper is organised as: Section 2 explains Related Work, Section 3 highlights Simulation Methodology and Environment, Section 4 gives Results 
and Discussions and at last Section 5 concludes the paper.

\section{RELATED WORK}

Several researchers have carried out analysis of different routing Protocols on the basis of $Q o S$ Parameters by performing simulations on different network simulators. In [2] the comparison of protocols namely AODV, DSR and AODV have been done by considering performance parameters like CBR (Constant Bit Rate) traffic on the basis of network throughput, data packet latency and packet delivery rate. Adhoc On demand Multipath distance vector routing (AOMDV) protocol has been proposed in [3] that uses queue length estimation technique in order to reduce the congestion in sending the data packets and RREQ. More specifically for reliable and efficient communication, a load balancing algorithm has been designed and implemented in this paper. In [4] the most efficient routing protocol has been selected among AODV, ADV and GOD for emergency situations in highway scenarios on the basis of performance metrics- network loss, transmission delay, throughput and collision rate. The reactive routing protocols namely AODV, DSR and AOMDV have been compared in [5] by changing parameters like node speed, mobility and sources that use various performance metrics. Two types of graphs were generated in mobility model like space graph and user defined. They concluded that AOMDV protocol has major drawback that it requires large routing load due to its degraded performance in space graph model.

In the earlier reported work, the focus is only on the evaluation of routing protocols. However in this work cross layer approach has been adopted by considering parameters of MAC Layer along with Network Layer. For this purpose queue size is considered as the most significant parameter. Considering this as a goal, this paper aims to find out optimum queue length for various routing protocols and hence determine the most efficient routing protocol on the basis of performance of QoS parameters and also compares the performance of MAC Interfaces.

\section{Simulation Methodology AND ENVIRONMENT}

For real time traffic environment, a platform is required for carrying both network and traffic simulations. NCTUns 6.0 (National Chiao Tung University) is a hybrid simulator and is based upon kernel re-entering methodology. It can simulate 802.11a, 802.11b, 802.11g, 802.11e technologies and uses TCP/IP stack for packets transmission [13].

\section{A. Performance Metrics}

The important performance parameters that have been identified in this work are:

- Packet Collision Rate: It implies the number of packets that collide per second. For optimum performance packet collision rate should be as low as possible.

- Packet Drop Rate: The number of packets that get dropped in network and as a result they are unable to reach the destination successfully defines the Packet Drop Rate. More packet drop rate deteriorates the performance of routing protocols.

- Broadcast Rate: It defines the total number of broadcasted packets successfully reaching the destination per unit time. More is the packet broadcast rate, better is the performance.

- Throughput Rate: It defines the total number of data packets successfully delivered at the destination node. It is calculated in NCTUns as "unpublished" [6] -

$$
T=\frac{N^{*} P_{s}}{T_{s}}
$$

where

$\mathrm{T}=$ Throughput

$\mathrm{N}=$ Number of packet delivered at destination

$\mathrm{P}_{\mathrm{S}}=$ Packet Size

$\mathrm{T}_{\mathrm{s}}=$ Total time

\section{B. Routing Protocols}

In this optimization work, routing protocols namely DSDV, AODV, ADV and GOD have been evaluated on the basis of aforementioned parameters.

\section{Simulation Scenario}

A simulation scenario has been created using 'Draw' feature of NCTUns for the selection of optimum queue size for various routing protocols. Multi interface car is used as a vehicle that moves on the road network. It supports various network interfaces out of which $802.11 \mathrm{a}$ interface has been used in this paper. The various parameters (of multi interface car and adhoc mobile nodes) are set using 'Edit' feature of NCTUns as shown in the following table.

Table 1. Simulation Parameters

\begin{tabular}{|c|c|}
\hline Parameters & Values \\
\hline MAC & $802.11 \mathrm{a}$ \\
\hline Routing Protocols & DSDV, AODV, ADV, GOD \\
\hline Queue Type & $10,25,50,75,100$ \\
\hline Queue Sizes(packets) & 15 \\
\hline Transmission Power(dbm) & 2400 \\
\hline Frequency(MHz) & 36 \\
\hline Speed (Kmph) & 11 \\
\hline Link Bandwidth(Mbps) & Two Ray Ground \\
\hline Path Loss Model & 30 \\
\hline Street Width(m) & Full Duplex \\
\hline Communication & stg, rtg \\
\hline Traffic Tool & 1 \\
\hline Antenna Gain(dpi) & 6.0 \\
\hline NCTUns Version & 400 \\
\hline Simulation time & \\
\hline
\end{tabular}


After parameters setting, the simulations are run using 'Run' feature available in NCTUns and results are obtained as shown in next section. The following figure shows the simulation scenario having wireless access points, adhoc mobile nodes, host computer and multi interface car equipped with 802.11a interface.

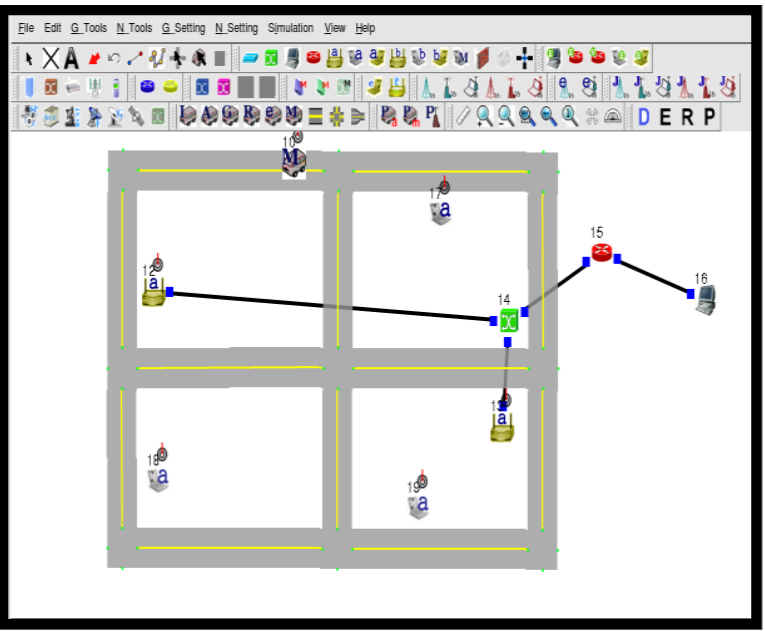

Fig.1. Simulation Scenario

\section{RESULTS AND DISCUSSION}

The graphs listed below shows the effect of changing queue length on performance of routing protocols. The graphs are based on following parameters:

- Packet Collision Rate

- Packet Drop rate

- Throughput Rate

- Broadcast Rate

\section{A. Simulative Investigation of DSDV Protocol}

The following graphs depict the results in the form of graphs of aforementioned performance parameters. The simulations are performed by considering five different queue lengths i.e. 10,25,50,75 and 100 packets. "Fig 2" depicts the packet Collision Rate in DSDV protocol at five queue sizes. It is determined from the graph that this protocol shows more no. of collisions when queue size is increased. At smaller queue lengths, number of collisions is comparatively lesser than that of larger queue sizes. "Fig 3" depicts the Packet Drop Rate at different queue lengths. Again with increase in queue length more number of packets is dropped and hence performance is degrades. "Fig 4" and "Fig 5" depicts the Throughput Rate and Broadcast Rate. It is evaluated that in this protocol maximum throughput rate is above 500 which is significant for 10,25 and 50 queue sizes whereas broadcast rate is maximum at queue length less than 50 packets i.e. more packets are broadcasted when queue size is less than 50 packets. Hence it is clear that in DSDV Protocol optimum results are obtained when queue size is less than 50 packets.

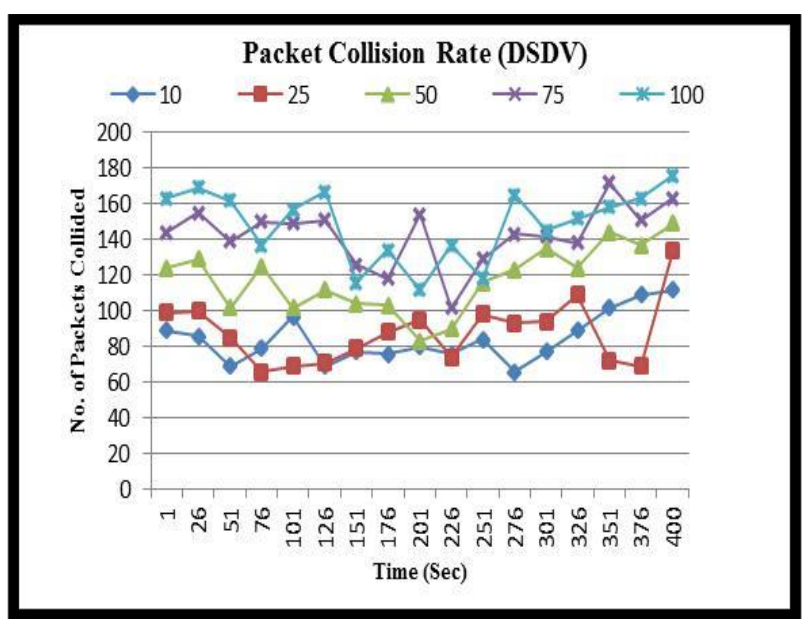

Fig.2. Packet Collision Rate using different queue sizes in DSDV Protocol

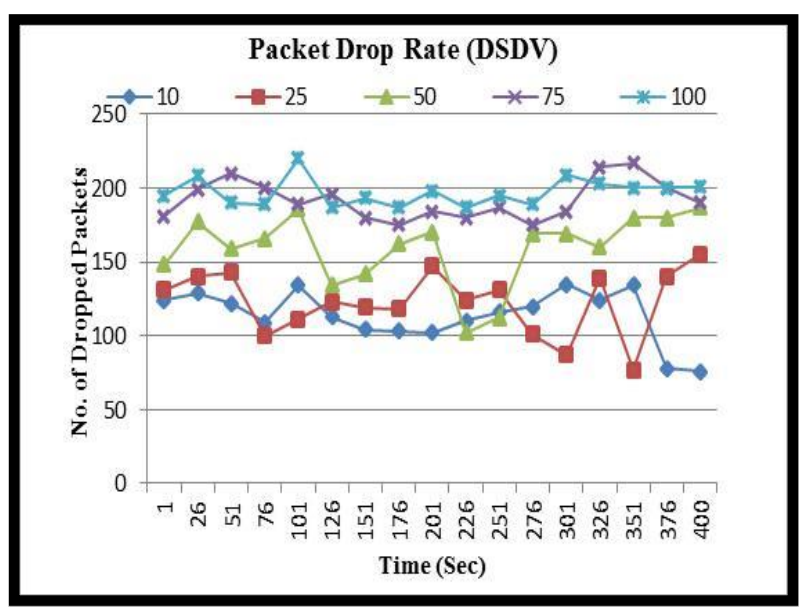

Fig.3. Packet Drop Rate using different queue sizes in DSDV Protocol

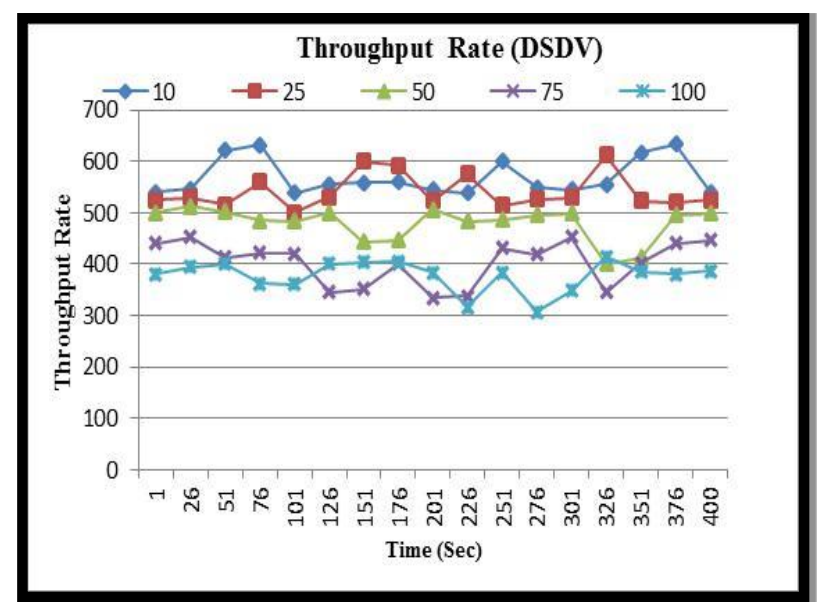

Fig.4. Throughput Rate using different queue sizes in DSDV Protocol 


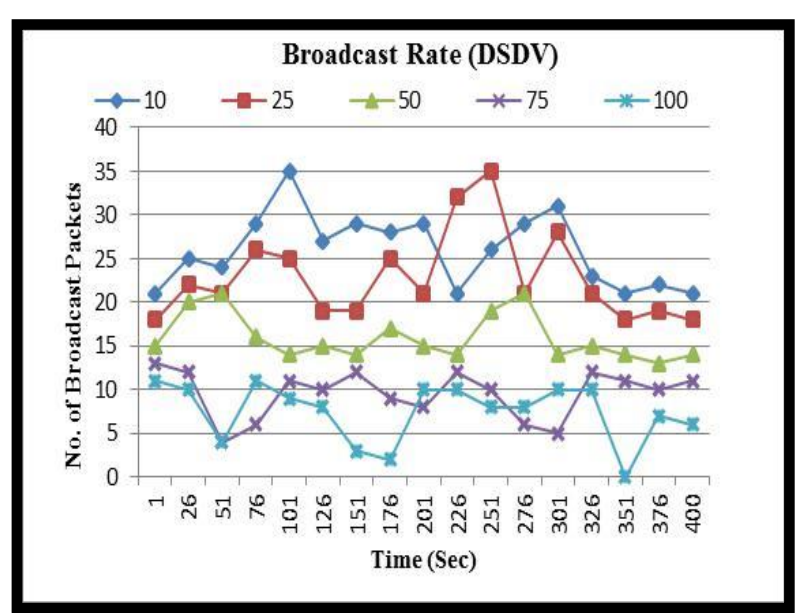

Fig.5. Broadcast Rate using different queue sizes in DSDV Protocol

\section{B. Simulative Investigation of AODV Protocol}

"Fig 6" depicts the packet collision rate in AODV Protocol. As AODV is a reactive routing protocol, its results are much better than DSDV. If we individually compare the no. of collisions at various queue sizes then it is evaluated that AODV has lesser no. of colliding packets. Here also with increase in queue size the no. of collisions increase and optimum results are obtained at smaller queue lengths. Further "Fig 7" depicts Packet Drop Rate, it is noticed that at smaller queue size the number of dropped packets are much less than larger queue size. Optimum results are obtained at queue length below 50 packets. At higher queue sizes, more packets are dropped. "Fig 8 " depicts throughput rate at different queue sizes. It is clear that optimum throughput values are obtained at queue lengths 50 packets and above. If we consider the average values it is clear that all queue lengths except for 100 packets length throughput rate is above 500 bytes/sec. "Fig 9" depicts the broadcast rate of data packets. It is evaluated that at smaller queue size more data packets are broadcasted successfully to the destination. Hence it is determined that for AODV Protocol, optimum queue length is below 50 data packets.

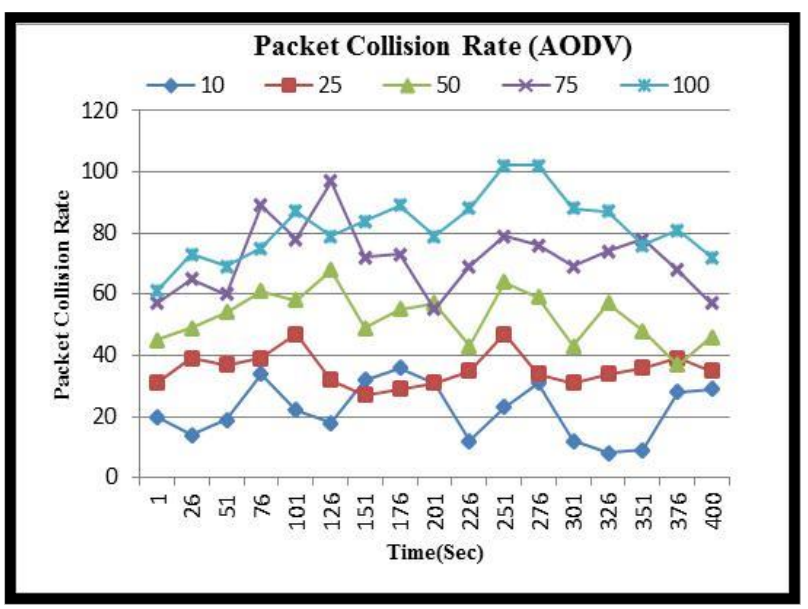

Fig.6. Packet Collision Rate using different queue sizes in AODV Protocol

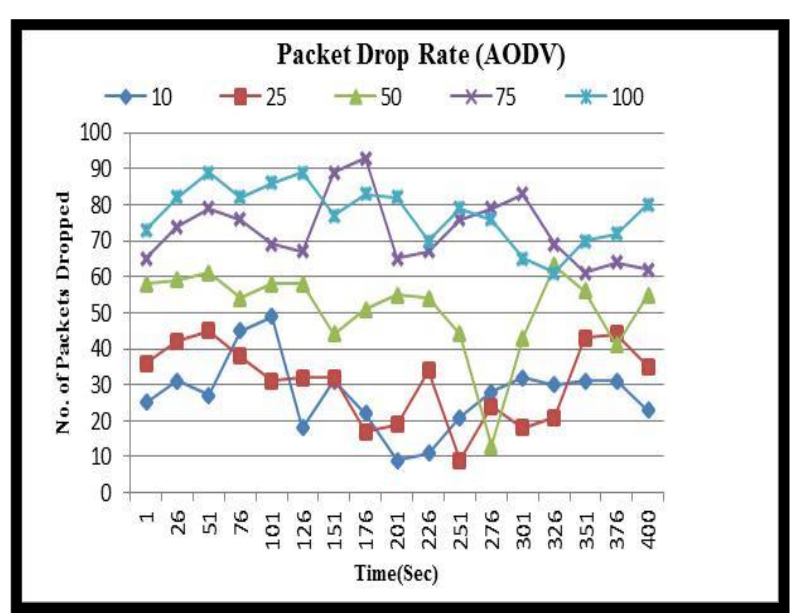

Fig.7. Packet Drop Rate using different queue sizes in AODV Protocol

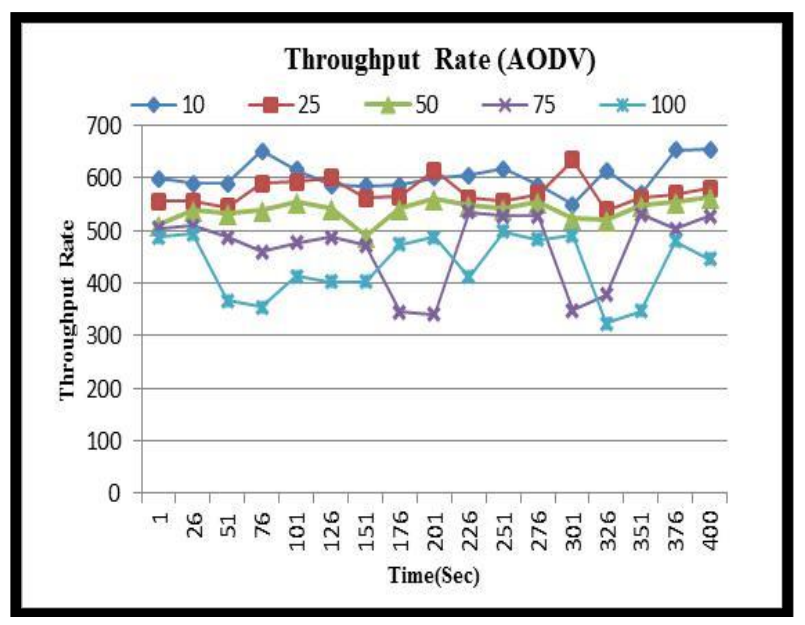

Fig.8. Throughput Rate using different queue sizes in AODV Protocol

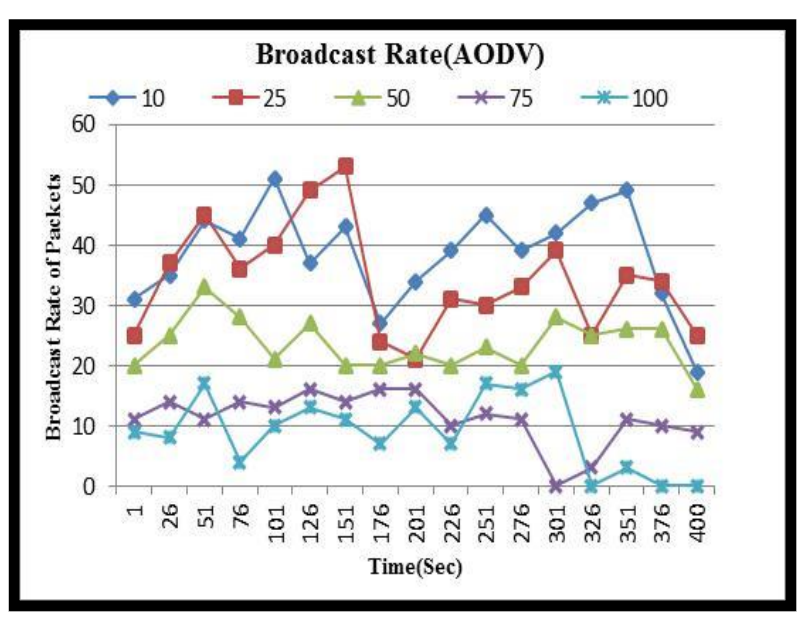

Fig.9. Broadcast Rate using different queue sizes in AODV Protocol

\section{Simulative Investigation of ADV Protocol}

"Fig. 10" depicts the packet collision rate in ADV Protocol. It is determined that being a hybrid routing protocol the results of this protocol are superior than any other protocol discussed so far. The no. of collisions is very less when queue size is below 50 packets. It is less among all the routing protocols discussed in this paper. "Fig 11" depicts packet drop rate. It is determined that no. of packet dropped are less than any other protocol and at 
queue size 50 and less than it lesser no. of packets are dropped. This implies that smaller queue sizes are more proficient. "Fig 12" and "Fig 13" depicts the Throughput rate and Broadcast rate. From both the graphs it is examined that smaller queue sizes are more effective than larger queue sizes.

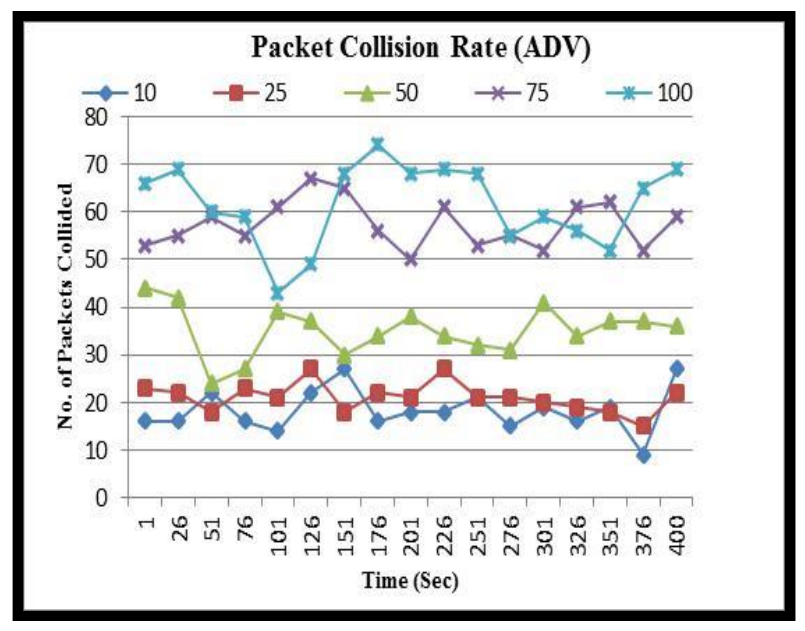

Fig.10. Packet Collision Rate using different queue sizes in ADV Protocol

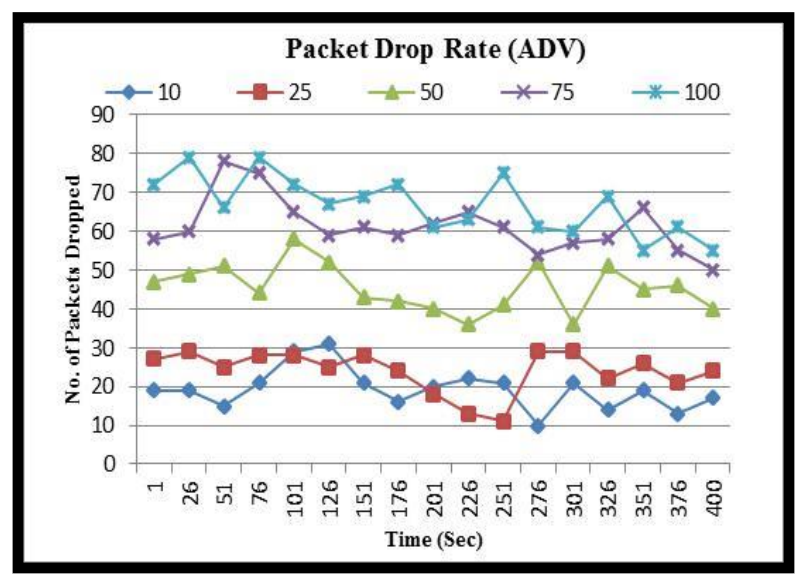

Fig.11. Packet Drop Rate using different queue sizes in ADV Protocol

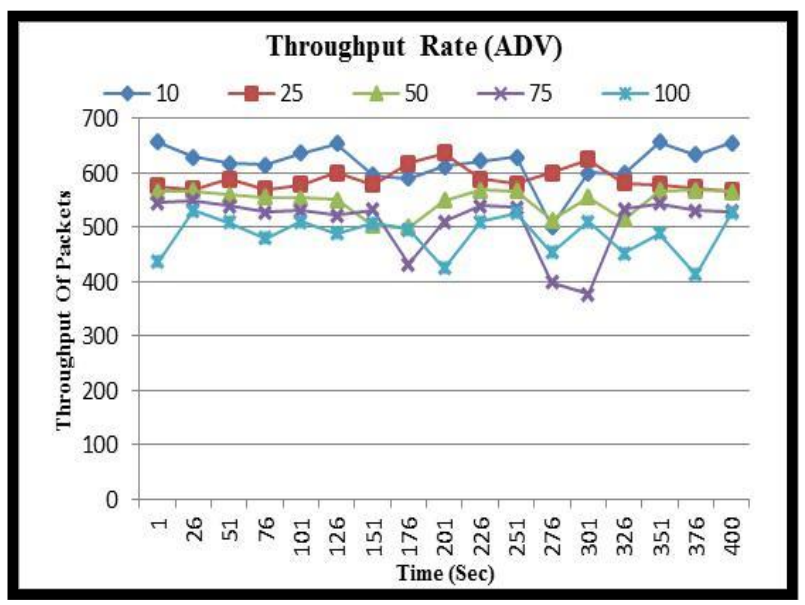

Fig.12. Throughput Rate using different queue sizes in ADV Protocol

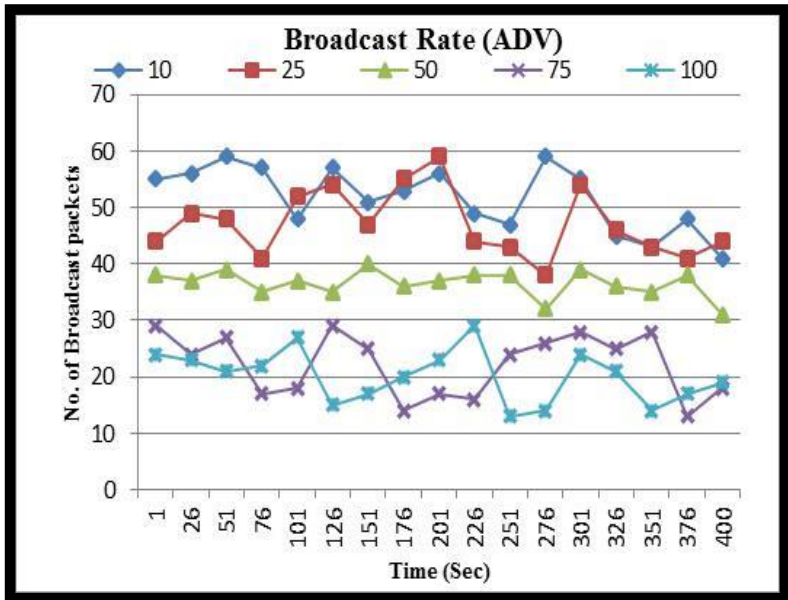

Fig.13. Broadcast Rate using different queue sizes in ADV Protocol

\section{Simulative Investigation of GOD}

In GOD the results produced are comparatively different from other protocols. In this protocol "Fig 14" depicts packet collision rate. It is determined that in this protocol, with increase in queue size from 10 to 25 packets no. of packet collisions increase whereas at 50 queue size the packet collisions are dropped to small value but with again increase in queue size, the packet collisions keeps on increasing vibrantly. "Fig 15" explains the packet drop rate. Here again at 50 packet queue length, no. of packets dropped are extremely less than at remaining queue sizes. Further "Fig 16" and "Fig 17" shows the Throughput Rate and Broadcast Rate. Again at 50 packet queue size more broadcast packets are reaching the destination and hence maximum is the throughput achieved.

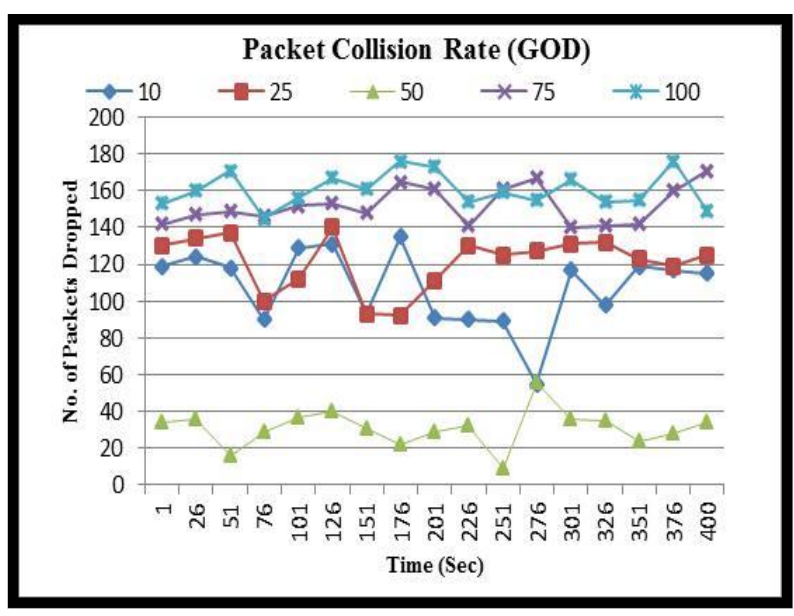

Fig.14. Packet Collision Rate using different queue sizes in GOD Protocol 


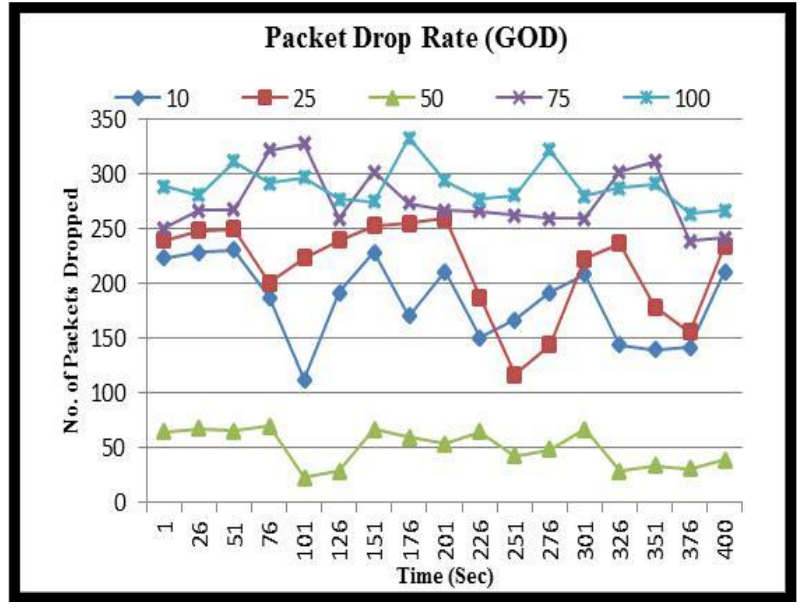

Fig.15. Packet Drop Rate using different queue sizes in GOD Protocol

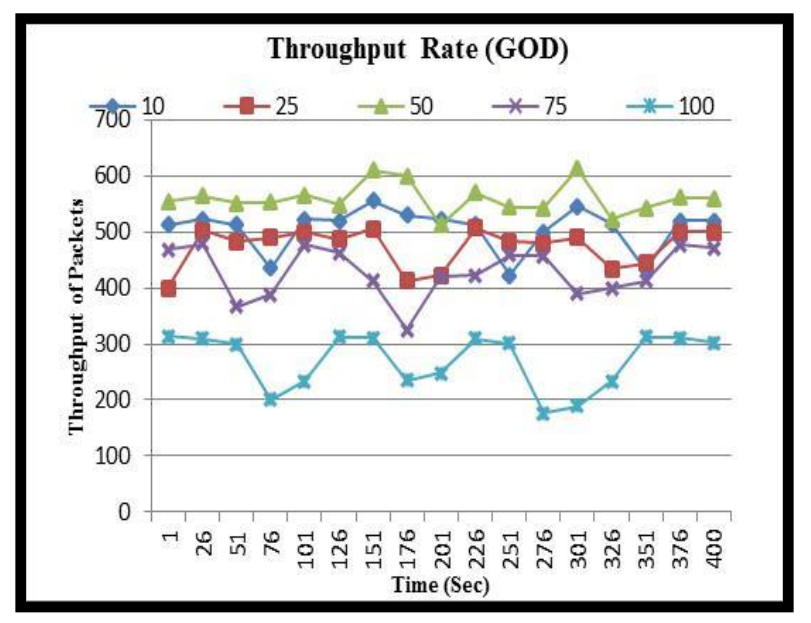

Fig.16. Throughput Rate using different queue sizes in GOD Protocol

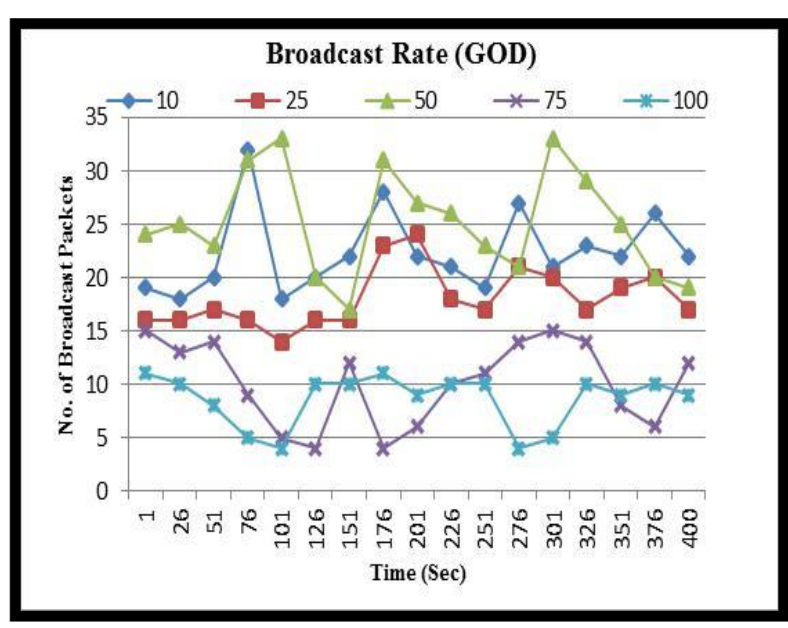

Fig.17. Broadcast Rate using different queue sizes in GOD Protocol

Following "Table 2" shows the comparison of average values obtained in routing protocols namely AODV, ADV, DSDV and GOD on the basis of QoS parameters at five different queue lengths.

Above "Table 2" depicts the performance of four routing protocols at 10,25, 5075 and 100 packets queue size in terms of QoS Parameters. After analysing the graphs and above table it is determined that all the routing protocols give better performance at small queue sizes except in GOD in which optimum results are obtained at 50 packet queue length. It is clear that in DSDV, AODV and ADV routing protocols, with increase in queue size the performance start degrading. As the queue size increases from 10 to 100 packets, the number of packet collisions per unit time, packet drop rate keeps on increasing gradually. Greater is the queue length more

Table 2. Comparative Performance of Routing Protocols v/s Queue Size

\begin{tabular}{|c|c|c|c|c|c|}
\hline \multicolumn{6}{|c|}{ Dynamic Routing Protocols } \\
\hline QoS Parameters & Queue Size & AODV & ADV & GOD & DSDV \\
\hline \multirow{5}{*}{$\begin{array}{c}\text { Packet Collision } \\
\text { Rate }\end{array}$} & 10 & 21.304 & 14.322 & 119.647 & 86.121 \\
\hline & 25 & 37.471 & 22.131 & 129.218 & 91.218 \\
\hline & 50 & 55.476 & 41.914 & 37.213 & 123.533 \\
\hline & 75 & 65.362 & 53.216 & 131.324 & 142.551 \\
\hline & 100 & 76.913 & 66.471 & 133.281 & 152.704 \\
\hline \multirow{5}{*}{ Packet Drop Rate } & 10 & 28.894 & 19.324 & 224.186 & 127.331 \\
\hline & 25 & 39.858 & 27.761 & 244.282 & 129.379 \\
\hline & 50 & 59.697 & 47.913 & 65.564 & 171.283 \\
\hline & 75 & 63.287 & 52.635 & 173.987 & 251.384 \\
\hline & 100 & 79.583 & 68.124 & 179.384 & 276.786 \\
\hline \multirow{5}{*}{ Throughput Rate } & 10 & 570.229 & 612.987 & 512.198 & 540.315 \\
\hline & 25 & 566.388 & 573.123 & 492.654 & 526.743 \\
\hline & 50 & 557.274 & 565.981 & 554.389 & 509.923 \\
\hline & 75 & 509.523 & 549.435 & 468.231 & 443.989 \\
\hline & 100 & 489.969 & 525.298 & 314.876 & 383.468 \\
\hline \multirow{5}{*}{ Broadcast Rate } & 10 & 28.516 & 54.231 & 19.422 & 21.209 \\
\hline & 25 & 25.305 & 43.890 & 15.209 & 18.295 \\
\hline & 50 & 23.772 & 36.927 & 24.398 & 15.305 \\
\hline & 75 & 17.243 & 28.984 & 12.894 & 13.878 \\
\hline & 100 & 16.124 & 21.456 & 11.823 & 12.845 \\
\hline
\end{tabular}


are the dropped packets and lesser is the throughput. With increase in queue length the delay for packets to reach the destination also increases. After carrying out extensive simulations and analysing the results of performance parameters it is determined that in DSDV, AODV and ADV optimum queue length is 10 packets whereas in
GOD optimum queue length is 50 data packets. Further results of AODV and ADV are considered to be efficient. At last after evaluating performance of all QoS parameters, ADV is chosen as the most efficient Routing protocol for this scenario.

Table 3. Comparison of MAC Interfaces

\begin{tabular}{|c|c|c|c|}
\hline \multicolumn{4}{|c|}{ Comparative Analysis of ADV Protocol in two MAC Interfaces } \\
\hline & & ADV Protocol in & ADV Protocol in \\
\hline QoS Parameters & Queue Size & 802.11a MAC & 802.11b MAC \\
\hline \multirow{5}{*}{$\begin{array}{c}\text { Packet Collision } \\
\text { Rate }\end{array}$} & 10 & 14.322 & 19.121 \\
\hline & 25 & 22.131 & 27.218 \\
\hline & 50 & 41.914 & 54.379 \\
\hline & 75 & 53.216 & 61.823 \\
\hline & 100 & 66.471 & 74.890 \\
\hline \multirow{5}{*}{ Packet Drop Rate } & 10 & 19.324 & 26.331 \\
\hline & 25 & 27.761 & 38.379 \\
\hline & 50 & 47.913 & 59.823 \\
\hline & 75 & 52.635 & 76.382 \\
\hline & 100 & 68.124 & 83.786 \\
\hline \multirow{5}{*}{ Throughput Rate } & 10 & 612.987 & 594.315 \\
\hline & 25 & 573.123 & 561.743 \\
\hline & 50 & 565.981 & 550.923 \\
\hline & 75 & 549.435 & 512.959 \\
\hline & 100 & 525.298 & 493.468 \\
\hline \multirow{5}{*}{ Broadcast Rate } & 10 & 54.231 & 45.219 \\
\hline & 25 & 43.890 & 37.291 \\
\hline & 50 & 36.927 & 26.301 \\
\hline & 75 & 28.984 & 22.878 \\
\hline & 100 & 21.456 & 15.834 \\
\hline
\end{tabular}

The above "Table 3" compares the performance of ADV Protocol in terms of QoS parameters on the basis of variable queue size in 802.11a and $802.11 \mathrm{~b}$ MAC Interfaces. Through simulative investigations in "unpublished" [14] ADV protocol has been chosen as the most efficient routing protocol on the basis of queue size. The above table compares the performance of ADV protocol in both MAC Interfaces. It is clear from the above table that ADV Protocol in 802.11a protocol gives more superior results than in 802.11 b. More specifically at the optimum queue size of 10 packets, the Packet Collision Rate in 802.11 b is $4.79 \%$ more than in $802.11 \mathrm{a}$; the Packet Drop Rate is 7\% less in 802.11a than 802.11bMAC. More is the packet drop rate; it results in degrading overall throughput of VANET. Hence the throughput rate is $18.67 \%$ greater in 802.11 a MAC than 802.11b. Although ADV Protocol gives optimum results in both MAC Interfaces but to evaluate the Interface in which more optimum results are produced work has been done in this paper. Hereafter analysing the performance of all QoS parameters we can say that ADV Protocol outperforms in 802.11a MAC than in 802.11bMAC.

\section{CONCLUSION}

In order to select the MAC Optimised Routing Protocol for VANET, work has been done in this paper. The goal of this paper is twofold. Firstly this paper investigates the optimum queue size for various routing protocols. After performing extensive simulations and examining the performance of QoS Parameters it has been evaluated that small queue size gives more optimum results. For AODV, ADV and DSDV 10 queue size gives comparatively better results than larger queue sizes whereas for GOD 50 packet queue size is chosen optimum. Secondly this paper investigates the optimised Routing protocols for MAC Interfaces. For 802.11a MAC $\mathrm{ADV}$ has been chosen as the most optimum routing protocol in terms of important QoS Parameters namely Packet Collision Rate, Packet Drop Rate, Throughput Rate and Broadcast Rate. At different queue sizes ADV Protocol gives more superior results than other protocols. For 802.11b MAC also in [14] ADV was chosen as the most efficient protocol in terms of QoS Parameters with an optimum queue size of 10 packets. By Comparing the performance of $\mathrm{ADV}$ in both MAC Interfaces it is determined that ADV outperforms in 802.11a MAC than 
802.11b MAC. Hence after investigating the simulation results it is found that ADV Protocol gives comparatively enhanced performance in 802.11a MAC than in 802.11b. The results produced in this paper are of great significance for the researchers working on queue size optimisation techniques.

\section{REFERENCES}

[1] M.S. Anwer, C. Guy, "A Survey of VANET Technologies", Journal of Emerging Trends in Computing and Information Sciences, Vol.5, No.9, 661-671, 2014.

[2] R. V. Boppana, S. P. Konduru, "An Adaptive Distance Vector Routing Algorithm for Mobile Ad Hoc Networks", IEEE INFOCOM , 1753-1762, 2001.

[3] A. Shukla, S. Sharma, "Queue Length based Load Balancing Technique using with AOMDV Protocol in MANET", International Journal of Scientific \& Engineering Research, Volume 4, Issue 10, 506-511, 2013.

[4] E. Jazayerifar, R. S. Nadooshan, "Performance Evaluation of AODV and ADV Routing Protocols in Safety Situations in Highway Environment", World appl. programming, Vol(5), No (8), 120-124, 2015.

[5] Shaikhul Islam Chowdhury, Won-Il Lee, Youn-Sang Choi, Guen-Young Kee, and Jae-Young Pyun, "Performance Evaluation of Reactive Routing Protocols in VANET,"17th Asia-Pacific Conference on Communications (APCC) IEEE 2011 pp. 559-564.

[6] Kanu Priya and Jyoteesh Malhotra, "Optimisation of MAC Layer to mitigate the effect of increased Mobility in VANET," accepted in Second International Conference Innovative Applications of Computational Intelligence on Power, Energy and Control with their impact on Humanity 18-19 November, 2016.

[7] K. Lee, U. Lee, and M. Gerla, "Survey of Routing Protocols in Vehicular Adhoc Networks," in Advances in Vehicular Ad-Hoc Networks: Developments and Challenges. Hershey, PA: IGI Global, 2009.

[8] B. Paul, "VANET Routing Protocols: Pros and Cons" International Journal of Computer Applications, vol. 20, no.3, 2011.

[9] T. Larson, N. Hedman, "Routing Protocols in Wireless Ad Hoc Network - A Simulation Study", Department of Computer Science and Electrical Engineering, Lulea University of Technology, Stockholm, 1998.

[10] R. Anggoro, "Performance Evaluation of AODV and AOMDV with Probabilistic Relay in VANET Environments," in Third International Conference on Networking and Computing, pp. 259-263, 2012.

[11] M. Abolhasan, T. Wysocki and E. Dutkiewicz, "A review of routing protocols for mobile ad hoc networks", Ad Hoc Networks, pp. 1-22, 2004.

[12] C. Perkins, E. Royer, "Ad-hoc on-demand Distance Vector Routing," Proc. 2nd IEEE Wksp. Mobile Comp. Sys. App., pp. 90-100, 1999.

[13] M. Abolhasan, T. Wysocki and E. Dutkiewicz, "A review of routing protocols for mobile ad hoc networks", Ad Hoc, pp. 1-22, 2004.

[14] Kanu Priya and Jyoteesh Malhotra, "On the Selection of Queue Optimised Routing Protocol in VANET," accepted in International Journal of Advanced Science and Technology, www.sersc.org/journals/IJAST, 2016.

[15] A GUI based user manual for NCTUns6.0 simulator.

\section{Authors' Profiles}

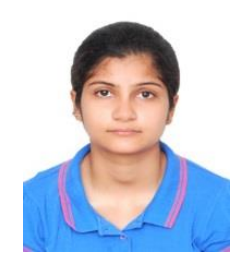

Kanu Priya was born in Jalandhar, Punjab, INDIA. She completed B.Tech degree with distinction in Computer Science and Engineering from DAV Institute of Engineering \& Technology, Jalandhar in 2014. Currently she is pursuing M.Tech degree in Computer Science and Engineering from GNDU Regional Campus, Jalandhar with specialization in Vehicular Ad-Hoc Network. Her research interests are in broad area of Wireless Communication and Vehicular Ad-hoc Networks.

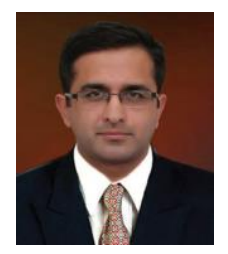

Dr. Jyoteesh Malhotra was born in Jalandhar, Punjab, INDIA. He completed B.Engg. with Distinction from P.R.M Institute of Technology \& Research, Amravati and M.Tech. with University Gold Medal from Guru Nanak Dev Engineering College, Ludhiana. He received $\mathrm{PhD}$ from Panjab Technical University in recognition to his contribution in the field of Wireless Communication \& Networks. From 1994 to 2007 he was employed with DAVCMC, New Delhi as Lecturer and Panjab University, Chandigarh as Assistant Professor. He joined GNDU Regional Campus at Jalandhar in July 2007 where he is currently Professor and Head of ECE \& CSE Departments.

His research interests are in the broad area of Pervasive Communication systems and Networks with emphasis on Statistical modeling of Fading Channels, Fading mitigation techniques, Optimization of High data rate Optical and wireless Communication Systems, Enhancement of QoS aware Wireless networks and Wireless Security. Dr. Malhotra has published and presented more than 170 technical papers in scientific journals and international conferences and authored 02 books. He is a life member of Indian Society for Technical Education (I.S.T.E.) and Editorial Board of many International Journals of repute.

How to cite this paper: Kanu Priya, Jyoteesh Malhotra,"On the Selection of MAC Optimised Routing Protocol for VANET", International Journal of Information Technology and Computer Science(IJITCS), Vol.9, No.2, pp.30-37, 2017. DOI: 10.5815/ijitcs.2017.02.04 